MATEC Web of Conferences 25, 01006

(2015)

DOI: $10.1051 /$ matec conf/ 20152501006

(c) Owned by the authors, published by EDP Sciences, 2015

\title{
Analysis of Tidal Prism Evolution and Characteristics of the Lingdingyang Bay at Pearl River Estuary
}

\author{
Shenguang Fang, Yufeng Xie \& Liqin Cui \\ Key laboratory of the Pearl River Estuarine Dynamics and Associated Process Regulation, Ministry of Water \\ Resources, Guangzhou, Guangdong, China
}

\begin{abstract}
Tidal prism is a rather sensitive factor of the estuarine ecological environment. The historical evolution of the Lingdingyang water area and its shoreline were analyzed. By using remote sensing data, the evolution of the water area of the bay was also calculated in the past 30 years. Due to reclamation, the water area was greatly decreased during that period, and the most serious decrease occurred between 1988 and 1995 . Through establishing the two-dimensional mathematical model of the Pearl River estuary, the tidal prism of the Lingdingyang bay has been calculated and analyzed. The hybrid finite analytic method of fully implicit scheme was adopted in the mathematical model's dispersion and calculation. The results were verified though the method of combining the field hydrographic data and empirical formula calculation. The results showed that the main tidal entrance of the bay is the Lingdingyang entrance, which accounts for about $87.7 \%$ of the total tidal prism, while Hong Kong's Anshidun waterway accounts for only $12.3 \%$ or so. Combining the numerical simulations and the historical evolution analysis of the water area and tidal prism, and compared with that in 1978, it showed that the tidal prism of the bay was greatly decreased, and the reduced area was mainly the inner Lingdingyang bay, which accounted for $88.4 \%$ of the whole shrunken areas. As weakening trends of the tidal powers of the medium and neap tides are obvious, the tidal prism is decreased during the medium and neap is larger than that during the spring.
\end{abstract}

Keywords: Lingdingyang Bay; tidal prism; empirical formula; shoreline; mathematical model

\section{INTRODUCTION}

The topography and geomorphology as well as hydrodynamic environment were gradually formed in the past thousands of years of evolution. The main three estuary types of the automatic adjustment and the geomorphological characteristics formation mechanism including the river dominant, the tide dominant and the wave dominant have been elaborated by $\mathrm{Li}^{[1]}$. It pointed out that, through factors contained in the system such as velocity, slope, water depth, estuary width and the composition of the sediment, the function of the estuary was constantly adjusted and formed some kinds of most optimal morphology to satisfy and meet the demand of energy input, output and transfer from two directions of the river and sea. Deposition, biological and chemical features are also important characteristic elements of the estuary because the diffusion and self-cleaning of the pollutants, ecological species and environmental mutations also have intrinsic connections with the estuarine system ${ }^{[2]}$. In recent years, the tidal flat reclamation and the construction of wading engineering such as port terminals have changed the estuary morphology sharply, leading to ecological wetland shrunk rapidly. According to statistics ${ }^{[3]}$, the area of land reclamation from the sea reached to 34 thousand $\mathrm{hm}^{2}$ in the Yangtze River estuary from 1978 to 2002. In the Yellow River Delta, due to beach reclamation, indiscriminate catching and sediment mining, the wetland wildlife resources were greatly reduced and the wetland environment was destroyed $^{[4]}$. In $1980 \mathrm{~s}$, due to the large area of wetland reclamation in Liaohe delta, the landform of the original wetland has been considerably changed, which substantially leads to the reduction of natural wetland area while artificial wetlands such as rice fields are gradually increased ${ }^{[5]}$. Human reclamation activities have not only directly caused the decrease of wetland area but also have caused the degradation of the wetland's habitat environment and the decline of biodiversity and ecological functions ${ }^{[6]}$. The fundamental reason is that the reclamation and the construction of estuary engineering and so on have changed the environmental condition of hydrology, hydrodynamics and sediment transportation. While sediment scouring and silting variations that caused by fluctuation of tidal channels and flow conditions in the estuary area often lead to great disasters. Therefore, it is necessary to do basic research on the pattern of the water and sediment movement characteristics and its natural adaptability, while weight and balance the reclamation and wetland protection ${ }^{[7]}$.

The shrink of intertidal zone and wetlands in the estuary area directly changed the sea topography and hydrodynamic boundary conditions, which also led to the change of tidal prism and water exchange in the bay. Tidal prism is an important index for the bay environment evaluation, which reflects the

This is an Open Access article distributed under the terms of the Creative Commons Attribution License 4.0, which permits unrestricted use, distribution, and reproduction in any medium, provided the original work is properly cited. 


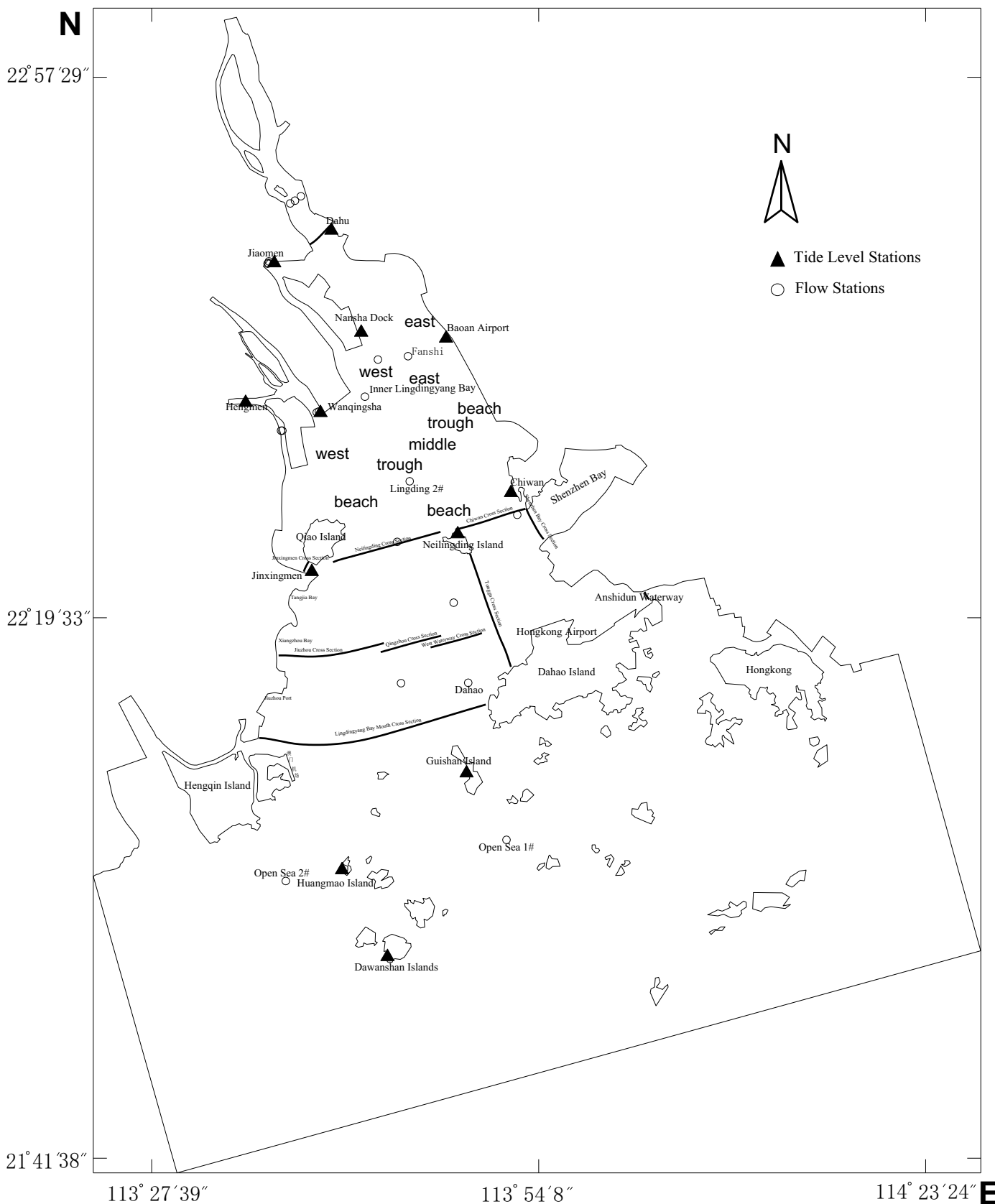

Figure 1. Sketch of the Lingdingyang bay

self-purification capacity of the bay and decides the exchange strength between the bay and the open sea, and also plays a significant role on the environment, ecology, sediment scouring and silting in the bay ${ }^{[8]}$. The ADCP (Acoustic Doppler Current Profiler) method was used to cruse and observe the Jiaozhou Bay estuary and obtain the tidal prism by Qiao ${ }^{[9]}$. Chen et al. ${ }^{10]}$ used both methods of charts and ADCP to calculate tidal prisms of the Jiaozhou Bay, and then compared and analyzed the results. Ye et al. ${ }^{[11]}$ separated intertidal zone and water area, calculated the tidal prism and water exchange time of Sansha Gulf. $\mathrm{Wu}$ et al. ${ }^{[12]}$ used remote sensing image data to obtain the water area of Haikou Port and then calculated the tidal prism changes. Considering the situation of reclamation in the estuary, Yang et al. ${ }^{[13]}$ improved the traditional calculation formula of tide, recalculated and analyzed the tidal prism of Jiaozhou Bay. 
In the recent thirty years, engineering constructions such as of sea reclamation, together with significant changes of the estuary shoreline as well as hydrologic and topographic conditions, have substantially affected the tidal prism and water exchange of the bay. Most research results ${ }^{[14][15][16][17][18]}$ are focused on the aspects of estuary hydrodynamics, water environment, water geomorphology and hydrology, which are closely and indirectly related to the tidal prism changes although still lack of fruits about it straightforwardly. Therefore, based on summarizing and analyzing the existing research results, combining with the hydrodynamic evolution and changes of water environment in the waters of the Pearl River estuary, this article discusses the process of the tidal prism evolution and the present condition of the estuary, adopting the method of combining historical data, empirical formula, field data and mathematical model.

\section{OVERVIEW ON WATER AREA AND SHORE- LINE EVOLUTION OF LINGDINGYANG BAY}

\subsection{Historical evolution of tidal water area}

As shown in Figure 1, the landform of the Lingdingyang bay generally maintained the pattern of "three beaches and two troughs" in the recent 100 years. The evolution of scouring and silting in the sea area is that the west beach is extending, the middle beach is expanding, the east beach is stable, and the west and eas troughs are narrowing ${ }^{[19][20]}$. Analysis to the topographic data and remote sensing image data of the Pearl River delta between 1966 and 1996 by Liu et al. ${ }^{[21]}$ shows the land area which is reclaimed during that period is $344 \mathrm{~km}^{2}$. The average annual land area which is reclaimed is $11 \mathrm{~km}^{2}$, most of which are distributed in the west bank of the bay, the Modaomen estuary area and the Huangmao coast. By using the GIS software, Chen et al. ${ }^{[15]}$ comprehensively analyzed the nautical chart and the remote sensing images of the Pearl River estuary in the past 20 years, that is, it was conducted from 1974 to 1997 . It shows that the beach area of the inner Lingdingyang waters which is shallower than 5 meters has shrunk $112 \mathrm{~km}^{2}$ during the past 23 years. The water area of the deep troughs which is deeper than 5 meters has generally shown a declining trend. Owning to the artificial maintenance dredging, it is only decreased by $24 \mathrm{~km}^{2}$ during the years. The overall area shrinking trend of the bay is very obvious. The water area was decreased by 136 $\mathrm{km}^{2}$ during the past 23 years. Especially from 1989 to 1997, it shrunk $109 \mathrm{~km}^{2}$ in past 8 years and it was annually decreased by $13.6 \mathrm{~km}^{2}$. Xu et al. ${ }^{[14]}$ shows that from 1953 to 1998, the area of inner Lingdingyang bay which is above theoretical depth datum is increased by about $175.34 \mathrm{~km}^{2}$, among which from 1974 to 1998 , it was increased by $112.6 \mathrm{~km}^{2}$, accounting for $64.2 \%$. It averages that continuous siltation and land reclamation have caused the water area to shrink constantly, which is the most prominent especially since 1990s. Simultaneously, deep trough area which is below 5 meters under the surface shows that the trend of constant was decreased by $19.6 \mathrm{~km}^{2}$ on total from 1974 to 1998 , and becoming narrower and deeper with the improvement of water and sediment transport capacity.

Comparing Chen et $\mathrm{al}^{[15]}$, $\mathrm{s}$ and $\mathrm{Xu}$ et $\mathrm{al}^{[14]}$, s data on the evolution of the shoal and trough in the Lingdingyang estuary, their results are basically the same, namely the area of inner Lingdingyang which is above 5 meters under the surface was respectively decreased by $112.0 \mathrm{~km}^{2}$ and $112.6 \mathrm{~km}^{2}$ from 1974 to 1998 . Deep trough areas of the elevations of sea bed which were below 5 meters from the surface have respectively decreased by $24.0 \mathrm{~km}^{2}$ and $19.6 \mathrm{~km}^{2}$ with a little difference between them.

\subsection{Historical evolution of Lingdingyang coastline}

The historical evolution of the Pearl River Delta can be described as the processes such as the sediment which is constantly piling up outside the estuary, the coastline which is constantly pushing towards the sea, the river course which is constantly extending, the estuary which is correspondingly moving outside and the branching bay which is gradually silting up. The artificial land reclaiming as well as the joint enclosing and floodgate building have accelerated the speed of the delta's deposition and the coastline's evolution over the recent 100 years. The related data analysis ${ }^{[21]}$ shows that most shores of the east coast of Lingdingyang bay have advanced 700 to 1,450 meters toward the sea during 30 years since 1966 . The average total advancing range is 1,330 meters, namely 44 meters a year. In the west coast, the shoreline deposition speeds of the west and north deltas, where the Jiaomen mouth, Hongqili mouth and Hengmen mouth are located and growing fairly fast. Take the ratio of the increased land area, which is the land reclaimed in this area, and the width of the delta's front line, the west and north deltas on the west coast of the bay have advanced $4.7 \mathrm{~km}$ towards the sea during 30 years since 1966, with an average about 156 meters a year. Xu et al.'s ${ }^{[14]}$ analysis on the coast line's changes of inner Lingdingyang bay between 1978 and 1999 shows that the coastlines on the west and east sides have shown the comprehensively advancing trend towards the sea. The main reasons are the renovation of Jiaomen mouth, Hongqili mouth and Hengmen mouth, as well as the land reclamation of Chiwan bay, Dachanwan bay and Jiaoyiwan bay on the east coast, especially changes on the west coast. Water areas in special years between 1978 and 1999 showed that the water area was decreased by $201.46 \mathrm{~km}^{2}$ during the 21 years due to the advancing shoreline with decreasing by $9.59 \mathrm{~km}^{2} \mathrm{a}$ year. According to Shen, et $\mathrm{al}^{[22]}$, the estuary's natural extension was the main trend of the Lingdingyang bay evolution before the 1980s. After that, it turned to the rapid extension of the estuary, which is mainly caused by the land reclamation. The water area of Lingdingyang bay has shrunk by about $22 \%$ during the 20 years 


\section{MATEC Web of Conferences}

but the general pattern of "three shoals and two troughs" keeps almost unchanged.

According to the results mentioned before, during the 20 years, namely from 1978 to 1999 , the land reclamation around the coast of Lingdingyang was the main cause of the constantly advancing coastline towards the sea, then it led to the rapid shrink of Lingdingyang's water area, and the advancing speed of the west coastline was much faster than the east.

\section{TIDAL PRISM EVOLUTION AND ANALYSIS OF THE LINGDINGYANG BAY}

\subsection{Calculation method of tidal prism}

Typically, the tidal prism refers to the volume of the seawater that a bay can take in on the average tidal range condition. The calculation formula is shown as follows:

$$
P=\Delta H \cdot S
$$

In this formula: $\mathrm{P}$ stands for the tidal prism on the average tidal condition; $\Delta H$ stands for the average tidal range; $\mathrm{S}$ stands for the average water area (i.e. the average value of the area is between the average high tidal level and the low tidal level). The difference between the average high tide level and the low tide level can be obtained according to the observed data of the tide station for many years. However, the calculation of $\mathrm{S}$ is more complex. Usually, only the 0 meter line (the theoretical lowest tidal elevation) and shoreline (the highest tidal elevation) are marked on the topography map, between which the average high tidal level and the low tidal level needed to be calculated. This article will calculate the historical evolution of the tidal prism at the Lingdingyang bay through the analysis of historical evolutions of its water area and shoreline.

\subsection{Water area of the Lingdingyang bay}

The water area calculations of the Lingdingyang bay in different periods are mainly based on remote sensing image data. The result of the statistics on the shoreline evolution of the Lingdingyang bay during the 29 years from 1978 to 2007 is shown in Table 1:

Table 1. Statistics of tidal prism evolution in the bay

\begin{tabular}{lll}
\hline \multirow{2}{*}{ Years } & $\begin{array}{l}\text { Decreased } \\
\text { water area }\end{array}$ & $\begin{array}{l}\text { Decreased ratio of } \\
\text { the tidal prism }\end{array}$ \\
\cline { 2 - 3 } $\mathrm{km}^{2}$ & $\%$ \\
\hline $1978 \sim 1988$ & 55.14 & 2.7 \\
$1988 \sim 1992$ & 61.09 & 3.0 \\
$1992 \sim 1995$ & 66.13 & 3.2 \\
$1995 \sim 1999$ & 23.9 & 1.2 \\
$1999 \sim 2003$ & 28.98 & 1.4 \\
$2003 \sim 2007$ & 18.6 & 0.9 \\
\hline Total & 253.83 & 12.3 \\
\hline
\end{tabular}

Analysis shows that because of the land reclama- tion(such as Hongkong Airport), the water area of the whole Lingdingyang bay was totally reduced by about $253.83 \mathrm{~km}^{2}$ during the 29 years with average decreasing rate of $8.75 \mathrm{~km}^{2}$ per year. The fastest period of reclamation was from 1988 to 1995 , in which the total reclamation area during the 7 years was about 127.22 $\mathrm{km}^{2}$, accounting for $50.1 \%$ of the total reclamation area during the 29 years, and leading to the $18.17 \mathrm{~km}^{2}$ reduction of water area per year. After 1995, the reclamation area in the Lingdingyang bay was reduced year by year. The total reclamation area that located at the west area of the west trough, including Jiaomen estuary, Wanqingsha, Hengmen estuary, west bank and Qi'ao island, is about $165.7 \mathrm{~km}^{2}$, accounting for $65.3 \%$ of the total reclamation area of the Lingdingyang bay. The total reclamation area is near the east area of the east trough, it includes Jiaoyi bay, east bank of Lingdingyang, Dachan bay, Chiwan bay and Shenzhen Bay, and it is about $71.8 \mathrm{~km}^{2}$, accounting for $28.3 \%$ of the total reclamation area of Lingdingyang waters. Therefore, the major reclamation area of the Lingdingyang bay is the waters near the west bank. According to Xu et $\mathrm{al}^{[14]}$, the water area of the inner Lingdingyang was reduced by about $201 \mathrm{~km}^{2}$ from 1978 to 2000 with total $17.7 \%$ reduction. Shen, et al ${ }^{[22]}$ show that the water area of Lingdingyang reduced by about $22 \%$ during the 20 years since 1980 . Calculations show that the water area of the Lingdingyang bay was reduced by about $206.3 \mathrm{~km}^{2}$ from 1978 to 1999 in the article; it's very similar to the results of different researchers.

\subsection{Tidal evolution of the Lingdingyang bay}

Besides, considering the water area of tidal prism, the changes of the tidal dynamics, namely the average tidal range, should also be considered. By comparing the relations between the field tidal range and tidal prism passing through the Humen mouth from 1978 to 1979 and from 1999 to 2001 , Xu et al ${ }^{[14]}$ showed that tidal prism was substantially decreased during medium and neap tides afterwards, while the tidal range and tidal prism basically unchanged when the tidal prism reaches $15000 \mathrm{~m}^{3} / \mathrm{s}$. It shows that the dynamical condition which keeps the tidal channel stable changes a little. As to the whole topographical features of Lingdingyang waters, although many land reclamation areas and the shoreline changed a lot, the topography of Lingdingyang waters basically keeps its original surrounding pattern. After the managing and dredging red lines of the Lingdingyang bay were marked out, the land reclamation areas are mainly concentrated on the shoal area near the west bank within the lines. The main tidal channels of the Lingdingyang bay, namely the west trough and the east trough, are slightly affected in the past 30 years, showing little change of the average fluctuation of the tidal ranges in the whole Lingdingyang waters.

According to the tidal prism calculation formula (1), when the average tidal range changes a little, the changes of the tidal prism are mainly reflected in the changes of the tidal areas. According to calculation, 

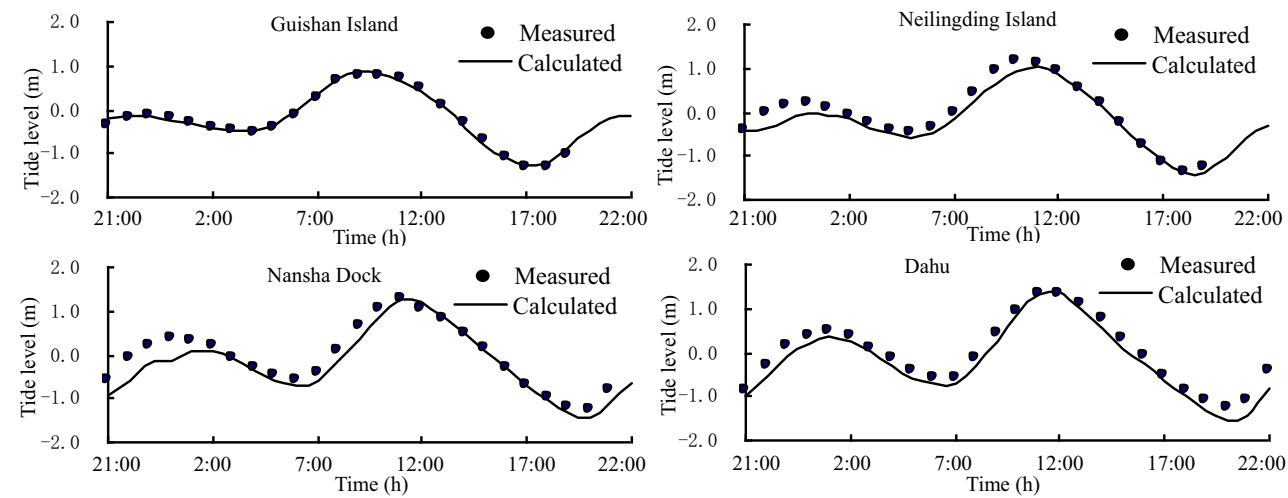

Figure 2. Water level verification curves at stations
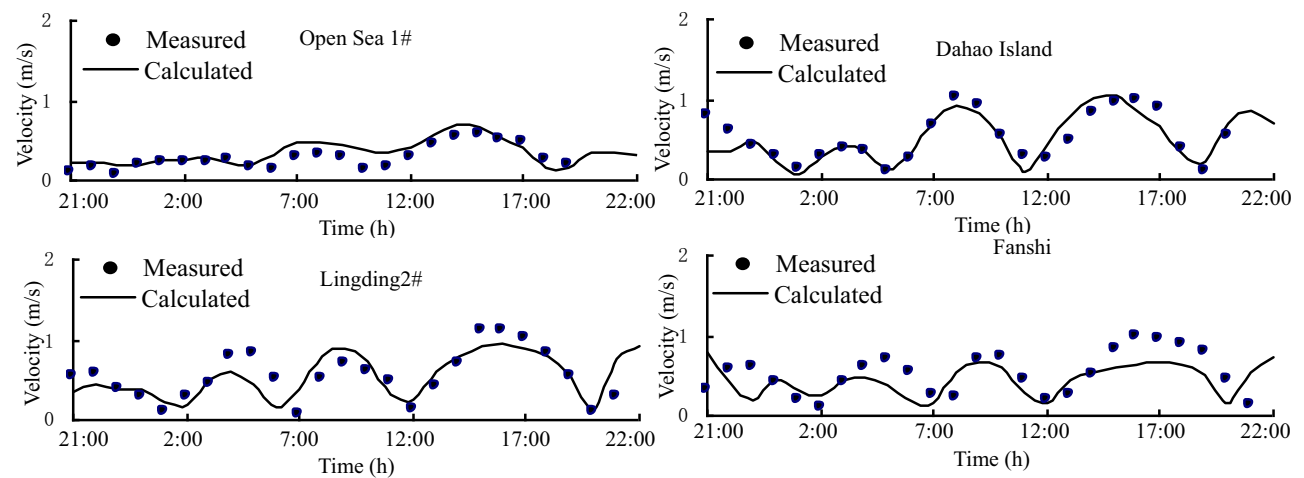

Figure 3. Velocity verification curves at stations

the current water area of the Lingdingyang bay is about $1,801.7 \mathrm{~km}^{2}$. It is totally reduced by $253.83 \mathrm{~km}^{2}$ from 1978 to 2007. So as shown in Table 1, the water area of the bay in 1978 was about $2,055.53 \mathrm{~km}^{2}$ according to the tidal prism reduction ratios which can be calculated in different periods compared with 1978 . It is obvious that the tidal prism of Lingdingyang waters has been reduced by about $12.3 \%$ in nearly 30 years since 1978 with $0.41 \%$ of average annual reduction.

\section{MATHEMATICAL MODELS OF THE LING- DINGYANG BAY}

\subsection{Mathematical model and scope for simulation}

The article adopts the two-dimensional hydrodynamic mathematical model of average water depth under the orthogonal curvilinear coordinates, which can be referred to the document ${ }^{[23]}$. The partially differential equation of two-dimensional mathematical model of tidal flow can be described as the following formation:

$E \frac{\partial \phi^{n+1}}{\partial t}+2 A \frac{\partial \phi^{n+1}}{\partial x}+2 B \frac{\partial \phi^{n+1}}{\partial y}=\frac{\partial^{2} \phi^{n+1}}{\partial x^{2}}+\frac{\partial^{2} \phi^{n+1}}{\partial y^{2}}+F$
The simulated water area of the Pearl River estuary is shown in Figure 1 with the maximum length being about $63 \mathrm{~km}$ from west to east and $145 \mathrm{~km}$ from north to south. The number of body-fitted orthogonal curvilinear grids is respectively adopted with $436 \times 665$ along $\xi$ and $\eta$ directions with the maximum grid size of $250 \mathrm{~m} \times 140 \mathrm{~m}$ and the minimum size of $30 \mathrm{~m}$ $\times 8 \mathrm{~m}$. The initial starting condition for numerical simulations is the stationary state. The four mouths in the upper boundary (Humen, Jiaomen, Hongqimen and Hengmen) are set as the field flux boundary. The tidal levels of the open seas' boundaries are set by field values through the interpolation method. The current speed adopts the second boundary conditions (Neumann boundary condition), namely the boundary's current speed of each time is set as the calculated value on the adjacently interior grid points. The shore boundary is set as the no-slip boundary condition.

\subsection{Model verification}

Here, it selected three tidal types to validate models which are the spring tide in August 13 to 14, 2007, the medium tide in August 16 to 17, 2007 and the neap tide in February 14 to 15,2001 . There are 12 stations 

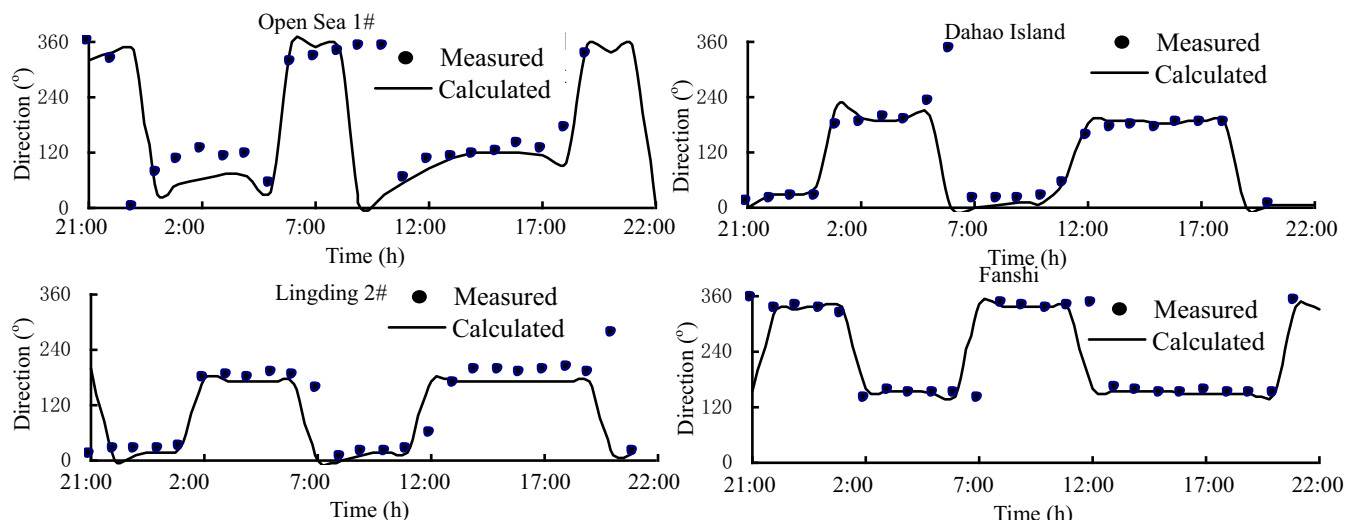

Figure 4. Direction verification curves at stations

Table 2. Tidal prisms calculated by measured data

\begin{tabular}{|c|c|c|c|c|c|c|c|}
\hline \multirow[t]{2}{*}{ Tide type } & \multirow[t]{2}{*}{ Stations } & $\begin{array}{l}\text { Avergae } \\
\text { high level }\end{array}$ & $\begin{array}{l}\text { Avergae } \\
\text { low level }\end{array}$ & $\begin{array}{l}\text { Average } \\
\text { water area }\end{array}$ & $\begin{array}{l}\text { Average } \\
\text { tidal range }\end{array}$ & $\begin{array}{l}\text { Tidal } \\
\text { prism }\end{array}$ & $\begin{array}{l}\text { Average tidal } \\
\text { prism }\end{array}$ \\
\hline & & $\mathrm{m}$ & $\mathrm{m}$ & $\mathrm{km}^{2}$ & $\mathrm{~m}$ & $10^{8} \mathrm{~m}^{3}$ & $10^{8} \mathrm{~m}^{3}$ \\
\hline \multirow{3}{*}{ Spring } & $\begin{array}{l}\text { Neilingding } \\
\text { Island }\end{array}$ & 0.66 & -0.95 & 1844.91 & 1.61 & 29.70 & \multirow{3}{*}{29.04} \\
\hline & Jingxingmen & 0.50 & -1.02 & 1840.07 & 1.52 & 27.97 & \\
\hline & Chiwan & 0.45 & -1.16 & 1830.07 & 1.61 & 29.46 & \\
\hline \multirow{3}{*}{ Middle } & $\begin{array}{l}\text { Neilingding } \\
\text { Island }\end{array}$ & 0.62 & -0.75 & 1853.69 & 1.37 & 25.40 & \multirow{3}{*}{25.27} \\
\hline & Jingxingmen & 0.54 & -0.77 & 1852.74 & 1.31 & 24.27 & \\
\hline & Chiwan & 0.42 & -1.00 & 1841.33 & 1.42 & 26.15 & \\
\hline \multirow{2}{*}{ Neap } & Jingxingmen & 0.48 & -0.51 & 1865.04 & 0.99 & 18.45 & \multirow{2}{*}{19.24} \\
\hline & Chiwan & 0.35 & -0.73 & 1853.30 & 1.08 & 20.02 & \\
\hline
\end{tabular}

used to validate the tide levels and 14 stations to valid the velocity and the flow direction. The verification curves of numerical results with field data of some stations (as shown in Figure 1, 4 tidal level stations and 4 tidal current stations) during the spring tide are shown in Figure 2, Figure 3 and Figure 4 with other detailed verification referred to the report ${ }^{[23]}$.

According to the model verification results, it appeared consistently with the field data regardless of water levels, velocities and flow directions, which showed the practicability of the mathematical model and its capability of simulating real tidal flow in the Lingdingyang bay.

\section{TIDAL PRISMS CALCULATED FROM MEASURED DATA}

By using the measured tidal levels during the spring tide and medium tide in 2007 and the neap tide in 2001, the tidal prism of the Lingdingyang bay can be calculated through the formula (1). According to stations in the bay, Neilingding Island, Chiwan and Jinxingmen stations are just located respectively in the middle of the bay. Therefore, the measured tidal levels of these three stations bear better representativeness to calculate tidal range. It shows that, including all shoals, the total water area of the Lingdingyang and Shizi- yang bays within the Pearl River estuary is about 1900 $\mathrm{km}^{2}$. Due to flood or ebb, the area of shoals is not the same, leading to the difference of tidal water areas. The high and low tidal levels and the tidal ranges of the typical tidal stations during the spring, medium and neap tides are shown in Table 2. According to the mathematical model, the average tidal areas from those corresponding to the different tidal levels were calculated. Then, adopting the tidal ranges, the tidal prisms during the tide types are calculated.

Results show that during the spring tide, the total tidal prism of the Lingdingyang bay is respectively $2.970,2.797$ and 2.946 billion $\mathrm{m}^{3}$, and the averaged tidal prism during the spring is 2.904 billion $\mathrm{m}^{3}$. During the medium tide, the total tidal prism is respectively $2.540,2.427$ and 2.615 billion $\mathrm{m}^{3}$ with the averaged tidal prism being 2.527 billion $\mathrm{m}^{3}$. During the neap tide in February 2001, the total tidal prism is respectively 1.883 and 2.002 billion $\mathrm{m}^{3}$ with the averaged tidal prism being 1.924 billion $\mathrm{m}^{3}$. The tidal prism during the spring tide is $13 \%$ larger than that during the middle tide, and during the middle tide, it is $23.9 \%$ larger than that during the neap tide. According to the results, the average water area is the largest during the neap, larger during the medium and the least during the spring. The main reason is that the exposed area of the shoal is very large during ebb in the spring, leading to the big reduced extent of the 
correspondingly average tidal waters area.

\section{ANALYSIS OF TIDAL PRISM CALCULATED BY THE MATHEMATICAL MODEL}

\subsection{Cross sections arranged for tidal prism}

Based on the mathematical model, cross sections are arranged in the estuary to record the inward and outward flux in different time to calculate the total tidal prism during one tidal current cycle. As shown in Figure 1, there are 11 characteristic cross-sections arranged in the Pearl River estuary named respectively by Lingdingyang bay mouth, Anshidun waterway, Jinxingmen, Neilingding, Chiwan, Shenzhen bay, Humen, Qingzhou, Jiuzhou, West Waterway and Tonggu. There are mainly two factors considered: on one hand, make sure to extract accurately from the results of the numerical simulations, so the arrangement of the sections must coincide with a grid line; on the other hand, be good for analyzing the characteristics of different water areas in the Lingdingyang bay.

\subsection{Tidal prism characteristics of the bay}

Based on the results by numerical simulation, flux in or out of the 7 main cross sections with time as well as the total tidal prism during a tidal cycle were recorded and shown in Table 3, Table 4 and Table 5 with the following characteristics.

(1)During a tidal cycle, the average tidal prisms of the Lingdingyang bay (sum of flux in and out of the Lingdingyang bay mouth and Anshidun waterway) in the spring, medium and neap tides were respectively $2.788,2.471$ and 1.953 billion $\mathrm{m}^{3}$. Compared with the tidal prisms shown in Table 2 and calculated based on formula (1), their differences are respectively $-77,-56$ and 29 million $\mathrm{m}^{3}$ with the error limited within $5 \%$. It verified the accuracy and effectiveness of the mathematical model and set the cross sections.

(2)During the spring, medium and neap tides, the average tidal prisms that passes through the Lingdingyang bay mouth and the Anshidun waterway sections respectively accounts for $87.7 \%$ and $12.3 \%$ of the total tidal prism of the Lingdingyang bay. Therefore, the main tidal entrance of the bay is the Lingdingyang bay mouth section. The tidal prism that passes through the Anshidun waterway section only accounts for a smaller proportion.

(3)Because of belonging to the type of irregular semidiurnal tide, the total tidal prism passing through the Lingdingyang bay mouth section during the first flood ( 1.983 billion $\left.\mathrm{m}^{3}\right)$ is greater than during the first ebb $\left(6.82\right.$ billion $\left.\mathrm{m}^{3}\right)$ while those during the second flood (3.091 billion $\left.\mathrm{m}^{3}\right)$ are significantly less than during the second ebb (4.021 billion $\left.\mathrm{m}^{3}\right)$ in a tidal current cycle of the spring. The same phenomenon was also existed in the medium and neap tides and more obvious under larger tidal range.

(4)The Neilingding cross section, between the Qiao island and the Neilingding island, is the main tide channel for the inner Lingdingyang bay with the average flux in and out of it, accounting for about $59.1 \%$ of the total tidal prism of the inner bay. The Chiwan section between the Neilingding Island and Chiwan bay is the second channel with its flux, accounting for about $28.8 \%$. While the Jinxingmen section is the smallest with the flux in and out of which accounts for about $12.1 \%$. The proportions of the inner Lingdingyang bay accepting seawater are respectively $58.2 \%$, $60.3 \%$ and $62.4 \%$ of the total tidal prism of the whole bay in the spring, medium and neap, showing the increasing trend with the decreasing tidal range and the average is about $60.3 \%$.

(5)The average proportion of the tidal prism that passes through the Humen section takes about $5.3 \%$ of the whole in the spring, medium and neap, which shows the slightly increasing trend along with the decreasing tidal range. It also indicates that the tidal prism of Shiziyang bay takes $5.3 \%$ of the total tidal prism. The average tidal prisms of Shenzhen bay were respectively 57, 44 and 28 million $\mathrm{m}^{3}$ in the spring, medium and neap, only accounting for average $1.8 \%$ of the total tidal prism.

(6)The averaged proportion of flux in and out of west waterway and Qingzhou cross sections takes about $46.6 \%$ of the total tidal prism of the Lingdingyang bay, indicating that the deep water area in the Lingdingyang bay entrance is the main tidal channel. The Jiuzhou cross section located at the shoal area only takes about $16.7 \%$ of the total tidal prism.

(7)The averaged proportion of flux in and out of Tonggu cross section takes about $12.2 \%$ of the total tidal prism, indicating that there is some tidal volume exchange between the east trough and the west trough through the section. The tidal prism of the section during flood is significantly greater than that during ebb, showing that part of the tidal prism exits from the Anshidun waterway cross section.

\subsection{Effect of the water area evolutions on tidal prism}

According to the above analysis of the historical data on the water area's evolution of the Lingdingyang bay, the water area is totally decreased by $253.83 \mathrm{~km}^{2}$ with tidal prism decreasing by about $12.3 \%$ during 30 years from 1978 to 2007 . The tidal prisms calculated by the field data during the spring, medium in 2007 and neap in 2001 are decreased by about $0.407,0.354$ and 0.270 billion $\mathrm{m}^{3}$ compared with 1978 . According to $\mathrm{Xu}$ et al. ${ }^{[14]}$, tidal dynamics of the medium and neap was decreased more by the decreasing water area of the bay, so the tidal prism in medium and neap is decreased more than that in spring. The substantial decrease of the tidal prism is one of the main reasons that cause the overall deterioration of the water ecological environment in the Lingdingyang bay.

From 1978 to 2007, the land reclamation mainly occurred within the inner Lingdingyang bay with the reclamation area reaching to $224.3 \mathrm{~km}^{2}$, accounting for $88.4 \%$ of the total reclamation in the whole Ling- 
MATEC Web of Conferences

Table 3. Tidal prisms in and out of cross sections at the Pearl River mouth

\begin{tabular}{|c|c|c|c|c|c|}
\hline \multirow{3}{*}{ Tidal type } & \multicolumn{2}{|c|}{ Lingdingyang bay mouth cross section } & \multicolumn{2}{|c|}{ Anshidun waterway cross section } & \multirow[b]{2}{*}{$\begin{array}{l}\text { Total } \\
\text { prism }\end{array}$} \\
\hline & Tidal prism & $\begin{array}{l}\text { Proportion of the total } \\
\text { tidal prism }\end{array}$ & Tidal prism & $\begin{array}{l}\text { Proportion of the } \\
\text { total tidal prism }\end{array}$ & \\
\hline & $10^{8} \mathrm{~m}^{3}$ & $\%$ & $10^{8} \mathrm{~m}^{3}$ & $\%$ & $10^{8} \mathrm{~m}^{3}$ \\
\hline Spring & 24.44 & 87.7 & 3.44 & 12.3 & 27.88 \\
\hline Medium & 21.68 & 87.7 & 3.03 & 12.3 & 24.71 \\
\hline Neap & 17.15 & 87.8 & 2.38 & 12.2 & 19.53 \\
\hline
\end{tabular}

Table 4. Tidal prisms of cross sections calculated by numerical simulation

\begin{tabular}{|c|c|c|c|c|c|c|c|}
\hline \multirow{3}{*}{ Cross section } & \multicolumn{2}{|l|}{ Spring } & \multicolumn{2}{|l|}{ Medium } & \multicolumn{2}{|l|}{ Neap } & \multirow{2}{*}{$\begin{array}{l}\text { Average } \\
\text { proportion } \\
\text { of the total } \\
\text { tidal prism }\end{array}$} \\
\hline & $\begin{array}{l}\text { Tidal } \\
\text { prism }\end{array}$ & $\begin{array}{l}\text { Proportion of } \\
\text { the total tidal } \\
\text { prism }\end{array}$ & $\begin{array}{l}\text { Tidal } \\
\text { prism }\end{array}$ & $\begin{array}{l}\text { Proportion of } \\
\text { the total tidal } \\
\text { prism }\end{array}$ & $\begin{array}{l}\text { Tidal } \\
\text { prism }\end{array}$ & $\begin{array}{l}\text { Proportion of } \\
\text { the total tidal } \\
\text { prism }\end{array}$ & \\
\hline & $10^{8} \mathrm{~m}^{3}$ & $\%$ & $10^{8} \mathrm{~m}^{3}$ & $\%$ & $10^{8} \mathrm{~m}^{3}$ & $\%$ & $\%$ \\
\hline Jinxingmen & 2.02 & 7.2 & 1.78 & 7.2 & 1.47 & 7.5 & 7.3 \\
\hline Neilingding & 9.51 & 34.1 & 8.82 & 35.7 & 7.25 & 37.1 & 35.6 \\
\hline Chiwan & 4.70 & 16.9 & 4.31 & 17.4 & 3.47 & 17.8 & 17.4 \\
\hline Shenzhen bay & 0.57 & 2.0 & 0.44 & 1.8 & 0.28 & 1.4 & 1.8 \\
\hline Humen & 1.44 & 5.2 & 1.30 & 5.3 & 1.08 & 5.5 & 5.3 \\
\hline Qingzhou & 5.14 & 18.4 & 4.70 & 19.0 & 3.85 & 19.7 & 19.0 \\
\hline Jiuzhou & 4.49 & 16.1 & 4.14 & 16.8 & 3.43 & 17.6 & 16.7 \\
\hline West waterway & 7.68 & 27.5 & 6.85 & 27.7 & 5.41 & 27.7 & 27.6 \\
\hline Tonggu & 3.33 & 11.9 & 3.04 & 12.3 & 2.41 & 12.3 & 12.2 \\
\hline
\end{tabular}

Table 5. Tidal prisms of the cross sections in the inner Lingdingyang bay

\begin{tabular}{|c|c|c|c|c|c|c|c|c|}
\hline \multirow{3}{*}{$\begin{array}{l}\text { Tidal } \\
\text { type }\end{array}$} & \multicolumn{2}{|c|}{ Jinxingmen } & \multicolumn{2}{|c|}{ Neilingding } & \multicolumn{2}{|c|}{ Chiwan } & \multirow{2}{*}{$\begin{array}{l}\text { Total tidal } \\
\text { prism of the } \\
\text { inner Ling- } \\
\text { dingyang } \\
\text { bay }\end{array}$} & \multirow{2}{*}{$\begin{array}{l}\text { Proportion of } \\
\text { the tidal prism } \\
\text { that the inner } \\
\text { Lingdingyang } \\
\text { takes }\end{array}$} \\
\hline & $\begin{array}{l}\text { Tidal } \\
\text { prism }\end{array}$ & $\begin{array}{l}\text { Proportion } \\
\text { of the total } \\
\text { tidal prism }\end{array}$ & $\begin{array}{l}\text { Tidal } \\
\text { prism }\end{array}$ & $\begin{array}{l}\text { Proportion } \\
\text { of the total } \\
\text { tidal prism } \\
\end{array}$ & $\begin{array}{l}\text { Tidal } \\
\text { prism }\end{array}$ & $\begin{array}{l}\text { Proportion } \\
\text { of the total } \\
\text { tidal prism }\end{array}$ & & \\
\hline & $10^{8} \mathrm{~m}^{3}$ & $\%$ & $10^{8} \mathrm{~m}^{3}$ & $\%$ & $10^{8} \mathrm{~m}^{3}$ & $\%$ & $10^{8} \mathrm{~m}^{3}$ & $\%$ \\
\hline Spring & 2.02 & 12.4 & 9.51 & 58.6 & 4.70 & 29.0 & 16.23 & 58.2 \\
\hline Mediun & 1.78 & 11.9 & 8.82 & 59.2 & 4.31 & 28.9 & 14.91 & 60.3 \\
\hline Neap & 1.47 & 12.1 & 7.25 & 59.5 & 3.47 & 28.5 & 12.19 & 62.4 \\
\hline
\end{tabular}

dingyang bay. It indicated that there was higher decreasing proportion of the tidal prism in the inner Lingdingyang bay than other water areas. According to the numerical simulations, the tidal prisms of the inner Lingdingyang bay during the spring, medium and neap tidal types were respectively $1.623,1.491$ and 1.219 billion $\mathrm{m}^{3}$, and they were decreased by about $0.36,0.313$ and 0.23 billion $\mathrm{m}^{3}$ compared with 1978. Owing to the "three shoals and two troughs" pattern, the reclamation area of the west shoal and east shoal is respectively $165.7 \mathrm{~km}^{2}$ and $71.8 \mathrm{~km}^{2}$, accounting for $65.3 \%$ and $28.3 \%$ of the total reclamation, and it also shows that the decreasing tidal prism mainly occurred in the inner Lindingyang bay, of which the west shoal took the main position.

\section{CONCLUSIONS}

The historical evolution of the water area and the shoreline of the Lingdingyang bay were analyzed with the calculated tidal prisms in recent years. From 1978 to 2007 , the water area of the bay was decreased by about $253.83 \mathrm{~km}^{2}$. The reclamation peak period was in the past 7 years from 1988 to 1995 with the reclamation area accounting for about half of the total reclamation area in the past 30 years and mainly occurring in the west shoal of the inner Lingdingyang bay, after which the reclamation area was decreased rapidly year by year. By using formula (1), tidal prisms were calculated based on the water areas and tidal ranges, showing that the tidal prism of the bay was decreased by about $12.3 \%$ in nearly 30 years since 1978 , with the average annually decreasing rate about $0.41 \%$.

The two-dimensional mathematical model was established to simulate flow of the Pearl River estuary. The validity and reliability of the mathematical model have been verified by comparing results from numerical simulations with field data of tidal levels, velocities and flow directions of the stations. The tidal prisms of the bay were respectively calculated to be $2.904,2.527$ and 1.924 billion $\mathrm{m}^{3}$ in the spring, medium and neap by using the measured hydrological data. The formula (1) and the difference were $-77,-56$ 
and 29 million $\mathrm{m}^{3}$ compared with results by numerical simulations with the error within 5\%. The main tidal entrance of the bay is the Lingdingyang bay mouth cross section (from Macau to Dahao Island), which accounts for about $87.7 \%$ of the total tidal prism and the Anshidun waterway cross section in Hongkong accounts for only $12.3 \%$ or so. The tidal prism of the inner Lingdingyang bay is larger than the outer Lingdingyang water which is closed to open sea, and it accounts for about $60.0 \%$ of the total tidal prism of the whole bay, while the outer one accounts for $40.0 \%$.

Combined the results of tidal prism evolution from calculations by the field data and formula with the numerical simulations by the mathematical model, it could be concluded that compared with 1978, the tidal prisms of the Lindingyang bay during the spring and medium in 2007 and the neap in 2001 were decreased by about $0.407,0.354$ and 0.27 billion $\mathrm{m}^{3}$ and the decreasing area mainly occurred in the inner Lingdingyang bay, which accounts for about $88.4 \%$ of the total flux decrease of the Lingdingyang water. The reclamation area of the west shoal in the inner Lingdingyang bay accounts for about $65.3 \%$ in total, leading to the most significantly decreasing tidal prism compared with other water areas. The reclamation activities in recent years have little effects on the tidal power of the spring, while significantly weakened the tidal ranges of the medium and neap. So there was more decreasing proportion of tidal prisms in medium and neap than in spring, and this is one of the main reasons that causes the overall deterioration of the water ecological environment in the Lingdingyang bay

\section{ACKNOWLEDGEMENT}

This research was financially supported by the National Natural Science Foundation of China (GN: 51109232) and the Project of Science and Technology in Guangzhou City (GN: 2012J2200076).

\section{REFERENCES}

[1] Li C C. 1997. On the Estuarine System and Its Automatic Adjustment. Acta Geographica Sinica (in Chinese) 52(4): 353-36.

[2] Dai Z J, Ren J. \& Zhou Z F. 2002. Research advance in definition and classification of estuaries. Journal of Oceanography in Taiwan Strait (in Chinese) 19(2):254-26

[3] Chen J Y. \& Chen S L. 2002. Impacts of the South to North Water Transfer Project on ecological environment at the Yangtze River. Water Resources Protection (in Chinese) (3):10-13.

[4] Chen W F, Zhou W Z. \& Si Y X. 2003. Crisis of Wetlands in the Yellow River Delta and its Protection. Journal of Agro-Environment Science (in Chinese) 22(4):499-502.

[5] Luo H Y, Huang F. \& Zhang Y Z. 2003. Space-time change of marsh wetland in Liaohe delta area and its ecological effect. Journal of Northeast Normal University (Natural Science Edition) (in Chinese) 35(2):100-105.

[6] Gu D Q, Zhao X T. \& Xia D X. 2003. A systemic analysis of the environmental pressure factors to the degradation of coastal wetlands in China. Acta Oceanologica Sinica (in Chinese) 25(1): 78-85.

[7] Chen J Y. \& Chen S L. 2002. Estuarine and Coastal Environment Changes and Resource Sustainable Utilization. Marine Geology \& Quaternary Geology (in Chinese) 22(2): 1-7

[8] Wang H, Chen P M. \& Jia X P, et al. 2008. Advance in the research on water exchange in the sea area. South China Fisheries Science (in Chinese) 4(2):75-80.

[9] Qiao G Y, Hua F. \& Fan B, et al. 2008. Tidal Water Capacity Computed From ADCP Measurement at Bay Mouth. Advances in Marine Science (in Chinese) 26(3):285-291.

[10]Chen H X, Hua F. \& Liu N, et al. 2009. Comparison Among Different Methods for Tidal Prism Calculation Neap Tide of Jiaozhou Bay in Autumn 2006 as an Expounded Example. Advances in Marine Science (in Chinese) 27(1):11-15

[11]Ye H T, Wang Y G \& Cao B. 2007. Tidal Prism of Sansha Bay and Its Water Exchange With The Open Sea. Journal of Hohai University (Natural Sciences) (in Chinese) 35(1):96-98

[12] Wu L Y, Sun Y X \& Wang Z X. 1997. Remote Sensing of The Tidal Influx in Haikou Port. Coastal Engineering (in Chinese) 16(2):1-5.

[13] Yang S L, Chen Q M. \& Zhu J, et al. 2003. Computation of Storage Capacity for Tidal Water of Semiclosed Bays Where The Inter-Tidal Zone Is Partly Embanked - Taking Jiaozhou Bay As An Example. Marine Sciences (in Chinese) 27(8):43-47.

[14]Xu F J, Zhu S K \& Wang H. 2004. Analysis of Hydrodynamic Environment of Lingdingyang Bay and Improvement Strategy. Pearl River (in Chinese) (1):11-14.

[15]Chen S S, Li X. \& Zou C Y, et al. 2001. An analysis of the changes and evolvements on the foreshore and deep channels of lingding bay in Pearl River estuary in past twenty years. Marine Science (in Chinese) 25(6):52-54.

[16]Lin Z H. \& Liang S H. 1996. A study on the tidal currents in the waters of the Pearl River mouth. Marine Science Bulletin (in Chinese) 15(2):11-22.

[17] Yang M L, Lin Q. \& Huang H H, et al. 2005. Distribution Feature of COD in the Waters of Pearl River Estuary. Marine Science Bulletin (in Chinese) 24(4):22-26.

[18]Ke D S, Guan Z B. \& Yu H S, et al. 2007. Environmental pollution and study trend in Pearl River Estuary. Marine Environmental Science (in Chinese) 20(5):488-491.

[19]Liu P R, Wen P \& Zhou Z F. The Analysis of Influencing Factor on the Lingdingyang Swale Development at the Pearl River Mouth. Journal of the Graduates Sun Yat-Sen University (Natural Sciences) (in Chinese) 20(1):32-38.

[20]Xu J L, Li Y X. \& Cai F X, et al. 1985. Evolution of shoal and though development of the Lingdingyang Bay at Pearl River Estuary. Beijing: Marine Press (in Chinese) 


\section{MATEC Web of Conferences}

[21]Liu Y F, Han M K. \& Wu L, et al. 1998. Recent Evolution of Outlets in Zhujiang River Delta and the Prospect for Land Reclamation. Acta Geographica Sinica (in Chinese) 53(6):492-500.

[22] Shen H K, Liu J Y. \& Chen X D, et al. 2006. Analysis of Flood Discharge at the Sea Mouth of the Pear River. Pearl River (in Chinese) (2):10-12.
[23]Fang S G. 2010. Research and Development of Software for Water Flow and Quality Mathematical Models - Research on Effect of the Gangzhuao Bridge project on Tidal Prism and Water Exchange in Pearl River Mouth. Guangzhou: Pearl River Hydraulic Research Institute (in Chinese). 